\title{
CONTROLS AND ALIGNMENT FOR THE UNIVERSITY OF MARYLAND ELECTRON RING*
}

\author{
M. Virgo $^{\dagger}$, R.A. Kishek, S. Bernal, H. Li, M. Walter, T.F. Godlove, M. Reiser, P.G. O’Shea \\ Institute for Research in Electronics and Applied Physics, College Park, MD 20742 \\ D. Kehne, FM Technologies, Inc., Fairfax, VA 22032
}

\begin{abstract}
The University of Maryland Electron Ring (UMER) [1] is a compact experiment that will be used to study the fundamental properties of space-charge dominated beams. Because of the small physical dimensions and weak fields involved, maintaining the beam's orbit is a difficult challenge. Accurate characterization of the magnets, careful alignment, and an effective control system are all critical to the project's success. In this report, the key challenges are outlined and the procedures that will be implemented to address them are explained. Existing portions of the system are described, and future plans are evaluated with computer simulation.
\end{abstract}

\section{INTRODUCTION}

UMER is a model of future generations of accelerators that will require space-charged dominated beams [2]. Figure 1 shows the ring, which is roughly $3 \mathrm{~m}$ in diameter and operates at $10 \mathrm{keV}$. It is desirable to study the evolution of the beam over the longest possible distance; when the ring is complete, 100 turns or more will be possible. The beam completes a circuit of the ring in about $200 \mathrm{~ns}$, so the lifetime of the beam is between $1 \mu \mathrm{s}$ and $1 \mathrm{~ms}$. The beam's average diameter is about $2 \mathrm{~cm}$, and it travels in a $5 \mathrm{~cm}$ pipe.

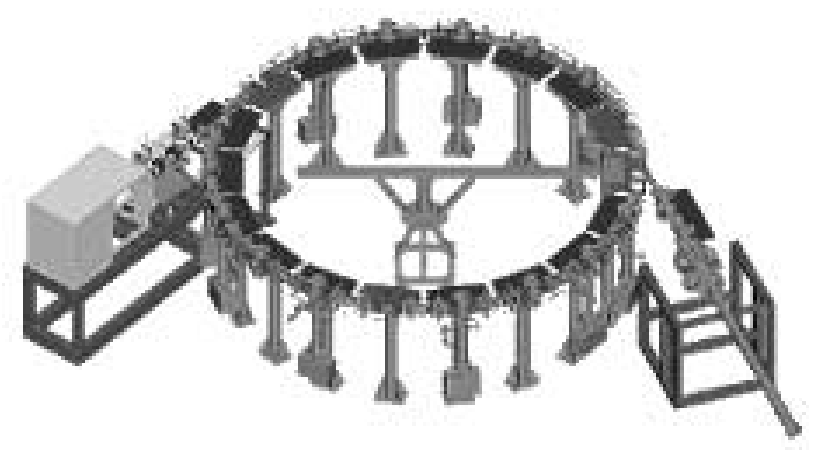

Figure 1: The UMER ring.

Despite its small scale, the ring shares much of the design complexity of larger accelerators. Specifically, 36 bending magnets and 72 focusing magnets are needed to confine the beam. As with all accelerators, preserving

\footnotetext{
* Work Supported by the U.S. Department of Energy

$\dagger$ mvirgo@glue.umd.edu
}

beam quality is of paramount importance. The specification for the ring is that $\Delta \epsilon / \epsilon \leq 4$ for 10 turns at full current and 100 turns at zero current. Several possible sources of emittance growth have been analyzed. For instance, magnet nonlinearities have been thoroughly characterized [3]. A study of the effect of quadrupole rotations is currently underway [4]. Another area of emphasis is very accurate injection [5].

Because the UMER beam is large, low energy, and extremely intense, image forces from the vacuum pipe are a significant cause of degradation of beam quality. In order to minimize this effect, it is necessary to steer the beam closely to its ideal orbit. This also reduces the impact of imperfections in the fields of the magnets, which tend to get worse far from their center. The design goal of the ring is to keep the rms centroid position within $1 \mathrm{~mm}$ of the axis. Although several factors, for instance dipole mispowering, contribute to steering error, exact placement of the lattice elements has proven to be the dominant (hardest to control) factor.

\section{ALIGNMENT}

A comprehensive study of tolerances was completed during the initial design of the ring [6]. Here, the analysis is updated to include hardware modifications and recent experience.

Alignment is accomplished using a 3D optical alignment system manufactured by Leica Geosystems. A pair of theodolites feed data to a computer that calculates the target's coordinates. A set of 9 monuments provide a fixed reference. The system is capable of resolving positions to less than $25 \mu \mathrm{m}$, but practical considerations will limit the resolution for UMER alignment to between $25 \mu \mathrm{m}$ and $.12 \mathrm{~mm}$.

Unlike most accelerator magnets, the ones used here are flexible printed circuits supported by aluminum mounts. They have a $2.8 \mathrm{~cm}$ radius and are $4.5 \mathrm{~cm}$ wide. Typical fields are on the order of $10 \mathrm{G}$. In fact, the fields are so small that the Earth's field helps steer the beam significantly. Figure 2 shows the magnets and their support structure. The rotation angle of a magnet mount is found by measuring two points in its plane of rotation, so the accuracy to which the rotation can be measured is set by both the accuracy to which each point can be measured and the distance between the points. In this case, the resolution of the rotation in radians is 40 times the resolution of the position in meters. The magnetic center of the magnet can be 


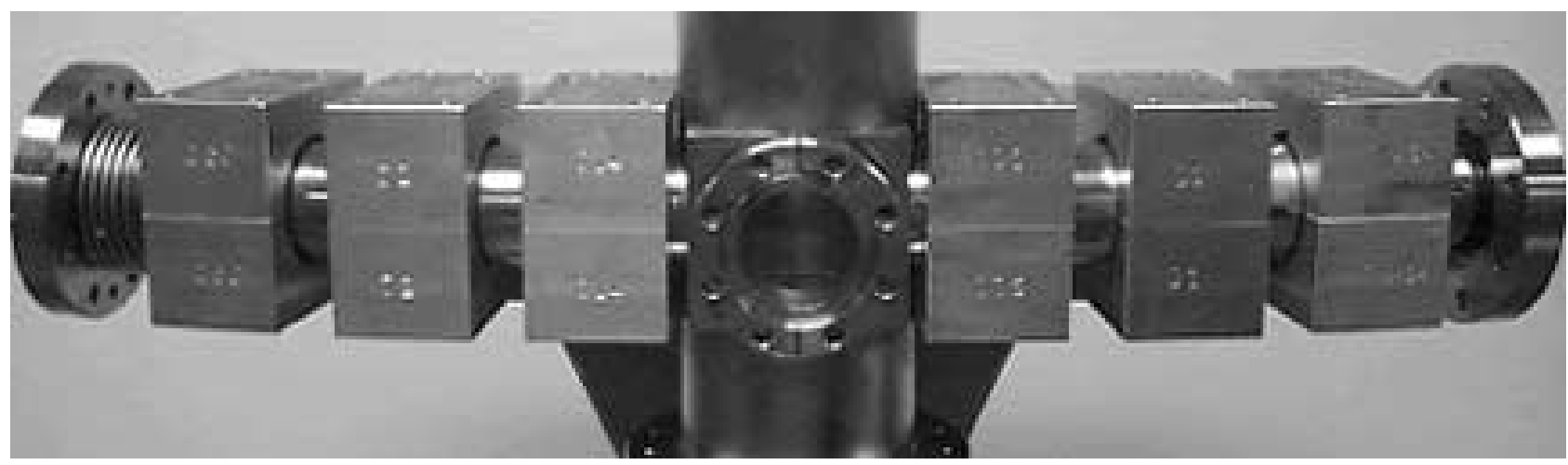

Figure 2: Two dipoles are each surrounded by two quadrupoles, comprising $1 / 18$ of the ring. The chamber in the center holds a BPM. The picture is about $.5 \mathrm{~m}$ across.

referenced to a point on the magnet mount with an accuracy of $.12 \mathrm{~mm}$. The rotation of the magnet with respect to the magnet mount is known to within $5 \mathrm{mrad}$.

The two types of magnet misalignment that directly affect the beam trajectory are dipole rotations and quadrupole displacements. The effect of these imperfections is analyzed below in the thin lens approximation. Because only centroid motion is of interest here, single particle techniques are used.

A rotated dipole steers the beam from the axis of the ring. This affects the trajectory of the beam in both the horizontal and vertical directions. Specifically, if a dipole is rotated by an angle $\phi$, the angle of the horizontal $(x /)$ and vertical $(y /)$ trajectories in the coordinate system of the perfect orbit are deflected by

$$
\begin{aligned}
\Delta x \prime & =\theta(1-\cos \phi) \\
\Delta y^{\prime} & =\theta \sin \phi
\end{aligned}
$$

where $\theta$ is the gross angle by which the beam is bent.

If the beam does not pass directly through the magnetic axis of a quadrupole, it will experience a net dipole force in addition to the focusing effect. The direction of the force depends on whether the displacement is in the focusing or defocusing axis of the magnet. If the quadrupole has a focal length of $\mathrm{f}$ and is displaced by $\Delta \mathrm{x}, \Delta \mathrm{y}$, the effect on the direction that the centroid will travel is

$$
\begin{aligned}
\Delta x \prime & = \pm(x-\Delta x) / f \\
\Delta y \prime & =\mp(y-\Delta y) / f
\end{aligned}
$$

\section{ERROR ACCUMULATION}

In order to investigate the accumulated error due to magnet misalignment, a simplified model of the ring is developed based on imperfect elements described by the equations above. The cumulative effect is numerically determined by iteration using Matlab. For each parameter set, 750 runs with independent sets of errors were generated. Each run consisted of 50 turns around the ring. Two statistics are recorded. The first is the rms centroid position. The second is the median value of the maximum centroid position for each run. This will indicate, for instance, whether or not the beam is likely to fit in the pipe. Figure 3 shows the results. The fixed error is included in all cases. Here, the phase advance was chosen to be far from any resonance $\left(85^{\circ}\right)$.

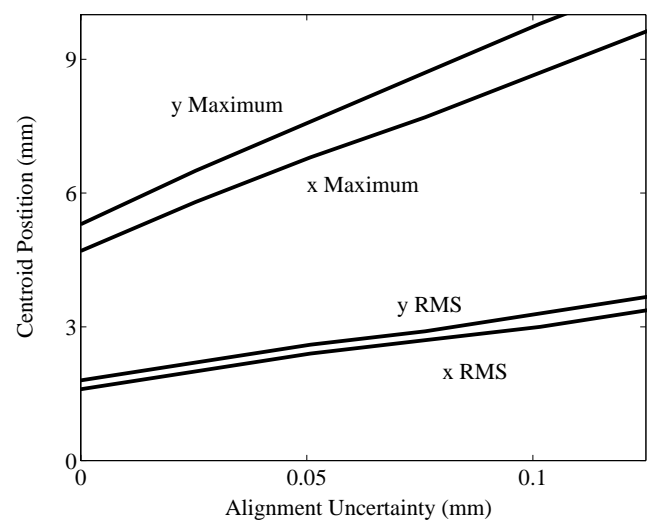

Figure 3: Predicted effect of misalignments on maximum and rms centroid position

Over the range being considered, a loosening of alignment tolerances leads to a linear increase in maximum and rms centroid position. The slight difference between the $\mathrm{x}$ and $\mathrm{y}$ axis is caused by the dipoles $(\sin \phi$ varies more rapidly than $\cos \phi$ for small $\phi$ ). The magnitude of the effect indicates that quadrupole displacements will dominate steering error due to misalignment. The data also indicates the requirement that the rms centroid position be restricted to less than $1 \mathrm{~mm}$ from the axis probably can not be met by accurate alignment alone. In order to achieve that goal, a correction mechanism is needed.

\section{CONTROLS}

Because UMER is purely a tool for understanding beam physics, there is great flexibility in the way the control system can be implemented. Beam position monitors (BPMs) 
are plentiful and well placed, as are correction magnets. Many different correction approaches can be tried without modifying the hardware.

Each of the 34 main bending dipoles and 34 correction dipoles are supplied by separate power sources set with an analog programming voltage and read through an analog voltage monitor. The voltages are accessed with VXI based D/A and A/D modules, which are computer controlled using National Instruments' LabVIEW software. Two pulsed magnets are needed; one to bend the beam into the ring and one to steer it out. These are controlled separately. Position data is provided by a set of 13 capacitive pickup BPMs [7]. The output of the BPMs is digitized with an oscilloscope and communicated to the computer through a GPIB interface.

A commonly described [9] correction algorithm has been evaluated with simulation [10]. The response of the beam to variations in the strength of a set of the dipoles is measured at each of the BPMs. The linear combination of magnet currents that centers the beam at each BPM is applied to the magnets.

The particle-in-cell code WARP [8] was used to follow the beam through 10 turns. Figure 4 shows the results for a representative run. In this case, there is a Gaussian distribution of quadrupole displacements with a standard deviation of $.2 \mathrm{~mm}$. No dipole rotation is included here, and 18 BPMs are used instead of the 13 available in the experiment. The rms centroid position has been reduced from $2.5 \mathrm{~mm}$ off center to $0.5 \mathrm{~mm}$, and the maximum distance the centroid strays from the axis has been reduced from $8.4 \mathrm{~mm}$ to $2.5 \mathrm{~mm}$.

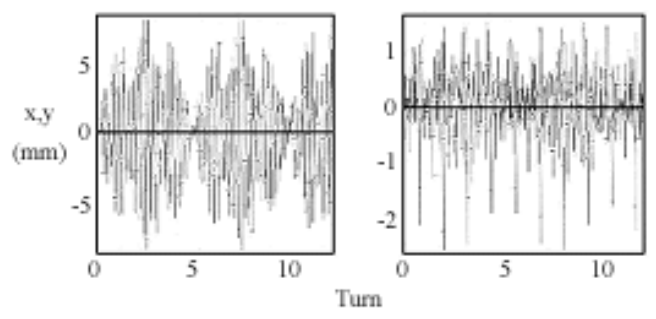

Figure 4: WARP simulation tracking centroid position as the beam makes 10 turns around the ring without (left) and with correction. Note the change in vertical scale

Other simulations have been carried out to study the effect of using fewer BPMs, the influence of imperfect dipoles, and the error in the position measured by the BPM. In most cases, an rms centroid position of less than $1 \mathrm{~mm}$ can be achieved using this method. In some extreme cases, the correction is not sufficient, and a more sophisticated algorithm may be necessary.

\section{CONCLUSIONS}

In this paper, the alignment approach and control mechanism for UMER have been described. A first order analysis of steering errors has been reported. It was found that accu- rate placement of the quadrupoles will be the fundamental test of the alignment process. It was also noted that a correction scheme is needed to meet the design requirement for rms centroid position.

An overview of the control system was given, and a basic correction technique based on that hardware was outlined. Simulation was used to evaluate the method. It was shown to mitigate the effects of alignment error well enough for proper operation of the ring under most circumstances. As the UMER construction progresses, the agreement between prediction and experiment will be closely monitored. Based on this experience, the simple model used here can be extended to meet the specific design requirements of future accelerators operating in the space-charge dominated regime.

\section{REFERENCES}

[1] http://www.ireap.umd.edu/umer

[2] P.G. O'Shea, et al., "The University of Maryland Electron Ring (UMER)," TOAA008, these proceedings.

[3] W.W. Zhang, et al., "Design and Field Measurements of Printed-Circuit Quadrupoles and Dipoles," Phys. Rev. ST Accel. Beams 3, 122401 (2000).

[4] H. Li, et al, "Printed Circuit Magnets for the University of Maryland Electron Ring - New Developments," TPPH060, these proceedings.

[5] S. Bernal, et al., "Beam Tests of the $10 \mathrm{keV}$ Injector for the University of Maryland Electron Ring (UMER)," WPAH023, these proceedings.

[6] L.G. Vorobiev, X. Wu, and R.C. York, "Single-Particle Beam Dynamics Studies for the University of Maryland Electron Ring,’ UMER Design Report, 1998 (unpublished).

[7] J. Harris, et al., "A Fast Beam Position Monitor for UMER," TPAH077, these proceedings.

[8] D. P. Grote et al., Fusion Eng. Des. 32-33, 193-200 (1996).

[9] S. Krinsky, in Handbook of Accelerator Physics and Engineering edited by A.W. Chao and M. Tigner (World Scientific, Singapore, 1999).

[10] Y. Li and R. Kishek, UMER Technical Note No. 99YL, 1999 (unpublished). 